\title{
RELAÇÃO ENTRE DESEMPENHO EDUCACIONAL E QUANTIDADE DE ALUNOS: UMA ANÁLISE EMPÍRICA REGIONAL COM DADOS DO SARESP
}

\author{
RELACIÓN ENTRE EL DESEMPEÑO EDUCACIONAL Y LA CANTIDAD DE \\ ALUMNOS: UN ANÁLISIS EMPÍRICA REGIONAL CON DATOS DEL SARESP
}

\author{
RELATIONSHIP BETWEEN EDUCATIONAL PERFORMANCE AND \\ STUDENTS QUANTITY: A REGIONAL EMPIRICAL ANALYSIS WITH DATA \\ OF THE SARESP
}

\author{
Camila Fernanda BASSETTO ${ }^{1}$ \\ Eric Chibana FERREIRA ${ }^{2}$ \\ Renato Forte AGUIAR ${ }^{3}$
}

RESUMO: O presente estudo contém uma investigação empírica acerca da existência, ou não, de uma relação entre o número de alunos nas escolas e o desempenho educacional em matemática. Escolas com maiores quantidades de alunos alcançam melhores resultados? Para responder tal questão, dados do SARESP, agrupados conforme as regiões do estado de São Paulo, foram utilizados para a realização de uma análise de correlação entre a quantidade de alunos por escola, que participaram da avaliação, e a média em matemática alcançada. Os coeficientes de correlação foram calculados e interpretados considerando informações referentes aos anos de 2008 e 2013. A partir dos gráficos de dispersão e dos coeficientes de correlação, elaborados e calculados a partir do software $\mathrm{R}$, associados ao número de alunos, que participaram das edições do SARESP de 2008 e 2013, e o desempenho em matemática, foi possível identificar que há regiões do Estado em que escolas com maiores quantidades de alunos obtiveram melhores desempenhos. Por outro lado, em outras regiões o coeficiente de correlação explicitou fraca relação tais variáveis. Dessa maneira, a mesma afirmação não pode ser mantida para todas as regiões nos dois anos considerados nesta pesquisa. A presença de características regionais e outras variáveis associadas à quantidade de alunos por escola e desempenho educacional podem exercer papel significativo na investigação por qualquer associação entre ambas variáveis.

PALAVRAS-CHAVE: Quantidade de alunos. Desempenho em matemática. SARESP. Análise de correlação.

RESUMEN: El presente estudio contiene una investigación empírica sobre la existencia, o no, de una relación entre el número de alumnos en las escuelas y el desempeño educacional en matemática. ¿Escuelas con mayores cantidades de alumnos

\footnotetext{
${ }^{1}$ Universidade Estadual Paulista (UNESP), Faculdade de Ciências e Letras, Araraquara - SP - Brasil. Professora Assistente Doutora no departamento de Ciências da Educação. E-mail: camila@ fclar.unesp.br. ${ }^{2}$ Universidade Estadual Paulista (UNESP), Faculdade de Ciências e Letras, Araraquara- SP - Brasil. Graduando em Administração Pública. E-mail: eric.chibana@hotmail.com.

${ }^{3}$ Universidade Estadual Paulista (UNESP), Faculdade de Ciências e Letras, Araraquara- SP -Brasil. Graduando em Administração Pública. E-mail: renato_faguiar@hotmail.com.
}

RIAEE - Revista Ibero-Americana de Estudos em Educação, Araraquara, v.12, n.4, p. 2072-2087, out./set. 2017. 
obtienen mejores resultados? Para responder tal cuestión, datos del SARESP, agrupados conforme las regiones del estado de Sao Paulo, fueron utilizados para la realización de un análisis de correlación entre la cantidad de alumnos por escuela, que participaron de la evaluación, y la media en matemática alcanzada. Los coeficientes de correlación fueron calculados e interpretados considerando informaciones referentes a los años de 2008 y 2013. Desde los gráficos de dispersión y los coeficientes de correlación, elaborados y calculados en el software $R$, asociados al número de alumnos, que participaron del SARESP en 2008 y 2013, y el desempeño en matemática, fue posible identificar que hay regiones del Estado en que las escuelas con mayores cantidades de alumnos obtuvieron mejores desempeños. Sin embargo, en otras regiones el coeficiente de correlación explicitó débil relación entre esas variables. Delante de eso, la misma afirmación no puede ser mantenida para todas las regiones en los dos años considerados en esa pesquisa. La presencia de características regionales y otras variables asociadas a la cantidad de alumnos por escuela y al desempeño educacional pueden tener un papel significativo en la investigación por cualquier asociación entre las variables.

PALABRAS CLAVE: Cantidad de alumnos. Desempeño en matemática. SARESP. Análisis de correlación.

ABSTRACT: This study contains an empirical investigation about the existence or otherwise of a relationship between the students number in schools and the educational performance in mathematics. Do schools with larger numbers of students achieve better results? To answer this question, we used data of the SARESP, grouped according to the regions of the state of São Paulo, to perform a correlation analysis between the number of students per school, who participated in the evaluation, and the average in mathematics achieved. The correlation coefficients were calculated and interpreted considering information referring to the years 2008 and 2013. From the scatter plots and correlation coefficients, computed using software $R$, associated to the number of student that participated of the evaluation in 2008 and 2013 and performance in mathematics, it was possible identify that are regions of the State in which schools with higher quantities of students performed better. On the other hand, in other regions the correlation coefficient explained a weak relation for such variable. Thus, the same assertion cannot be maintained for all regions in the two years considered in this research. The presence of regional characteristics and other variables associated with the number of students per school and educational performance can play a significant role in the investigation by all association between both variables.

KEYWORDS: Quantity of students. Performance in mathematics. SARESP. Analysis of correlation.

\section{Introdução}

Compreender os aspectos determinantes da qualidade educacional é uma tarefa árdua. $\mathrm{O}$ efeito do tamanho da sala de aula sobre o desempenho do aluno tem sido um tema de discussão para educadores e está no centro de debates políticos focados na qualidade educacional e na alocação de recursos. Geralmente, quando questionados 
sobre o número de alunos em sala de aula, pais e professores se mostram favoráveis a classes menores, talvez porque acreditam que isto favoreça o aprendizado do aluno ou porque salas reduzidas ofereçam um ambiente mais agradável para os alunos e professores que nelas estão. Embora a quantidade de alunos em classe seja considerada uma variável fácil de ser manipulada quando comparada a outros fatores escolares, a dificuldade em mensurar o efeito exercido sobre a proficiência do aluno é significativa. Embora o nível educacional varie substancialmente entre e dentro das escolas, essas diferenças são frequentemente associadas a fatores como status socioeconômico do aluno. Provavelmente, por esta razão, muitas pesquisas sobre a relação entre tamanho da classe e desempenho é inconclusiva.

O processo educacional no Brasil pode ser descrito como atrasado, mesmo quando comparado a países menos desenvolvidos, além de altamente assimétrico em favor de uma fatia privilegiada da população. Desde a década de 80 têm ocorrido diversas mudanças institucionais incluindo políticas educacionais para reduzir a desigualdade, expandir o acesso a escola e melhorar as taxas de alfabetização. Mas somente em meados da década de 90, após um avanço educacional muito lento comparado a outros países, causado pela elevada repetência e falta de políticas educacionais apropriadas, é que o Brasil começou a ampliar o acesso à educação de forma relativamente rápida, conseguindo aumentar significativamente a frequência escolar em todos os níveis.

O sistema educacional brasileiro é sempre avaliado e, muitas vezes, apontado como um dos fatores responsáveis pelo baixo grau de desenvolvimento do país. $\mathrm{Na}$ década de 1990, a avaliação educacional começou a ter um papel de destaque nas políticas públicas, na busca por melhorias dos sistemas de avaliação implantados, e também visando responder e justificar o financiamento das políticas educacionais. Nos últimos anos, é perceptível o avanço obtido na área educacional considerando os recursos metodológicos disponíveis e os atuais critérios de avaliação, tais como o Sistema Nacional de Avaliação da Educação Básica (SAEB), o Sistema de Avaliação do Rendimento Escolar do Estado de São Paulo (SARESP), o Exame Nacional do Ensino Médio (ENEM), o Exame Nacional de Desempenho dos Estudantes (ENADE), e outros.

Os sistemas de avaliação têm revelado um quadro crítico para a educação formal em relação ao desempenho e rendimento escolar do indivíduo. Para obter melhorias na qualidade do ensino e alcançar um sistema educacional eficaz, no qual o aluno aprenda, seja aprovado e conclua a educação básica dotado da capacidade de armazenar, 
transformar e aplicar o conhecimento, é necessário que os responsáveis pelos processos de avaliação educacional entendam, decodifiquem e interpretem, à luz da realidade, os resultados dessas avaliações, explicitando a verdadeira deficiência evidenciada pelo aluno na avaliação de suas capacidades cognitivas.

A fim de contribuir com a discussão presente na literatura referente aos efeitos do tamanho da sala sobre o desempenho do aluno, o presente estudo busca verificar a existência de correlação entre o número de alunos por escola que participaram das avaliações e a pontuação média obtida em matemática nas edições do SARESP de 2008 e 2013 considerando alunos da terceira série do Ensino Médio de escolas públicas estaduais, agrupadas conforme as regiões do estado de São Paulo.

O artigo está estruturado em seções incluindo esta introdução. Na seção dois são apresentados os principais aspectos do SARESP. A seção três contém uma breve revisão de literatura destacando estudos realizados considerando o número de alunos em sala de aula. Na terceira seção é realizada uma análise empírica de correlação entre as variáveis "número e alunos por escola que fizeram a prova" e "pontuação média em matemática" e, finalmente, na seção quatro são apontados os resultados desta pesquisa.

\section{O Sistema de Avaliação de Rendimento Escolar do Estado de São Paulo - SARESP}

O SARESP surge com a finalidade de atender melhor as avaliações que, até então, são de caráter mais pontual. Passou a vigorar em 1996 e, atualmente, é aplicado nos $3^{\circ}, 5^{\circ}, 7^{\circ}$ e $9^{\circ}$ anos do Ensino Fundamental e na $3^{\text {a }}$ série do Ensino Médio. Utilizase de dois instrumentos de avaliação, sendo o primeiro caracterizado pela aplicação de provas em dois dias, para analisar o desempenho dos alunos do Ensino Fundamental e Médio em questões envolvendo leitura/escrita e matemática, e o segundo instrumento são questionários aplicados aos alunos, pais dos alunos e escolas (professor coordenador, professores das disciplinas, diretor) por meio do qual são extraídas informações sobre características pessoais, contexto socioeconômico e cultural, trajetória escolar, projeto pedagógico, corpo docente, entre outras.

Questões dessa natureza devem ser consideradas, uma vez que as desigualdades sociais têm implicações diretas sobre a educação, como apontam os estudos de Barbosa e Fernandes (2001), Jesus e Laros (2004) e Soares e Collares (2006). Jesus e Laros (2004) destacam a repetência, a evasão e o atraso escolar ao considerar a trajetória do aluno. De acordo com Laros et al. (2010), o desempenho escolar sofre, também, 
influência de fatores relacionados com elementos e traços que o aluno traz consigo e com o que a escola lhe oferece em termos de ensino, instalações e ambiente. Para Soares e Collares (2006), os melhores rendimentos são apresentados por alunos com níveis socioeconômicos mais altos.

O resultado do desempenho dos alunos descreve aquilo que os mesmos são capazes de fazer em relação às habilidades e competências avaliadas, conforme Matriz de Referência para Avaliação de Matemática do SARESP (BRASIL, 2011), na qual as competências $^{4}$ são reunidas em grupos, tais como 'competências para observar', 'competências para realizar' e 'competências para compreender'. A partir das expectativas de aprendizagem quanto ao conteúdo, competências e habilidades, estabelecidas para cada ano/série da disciplina no Currículo do Estado de São Paulo, os pontos da escala do SARESP são agrupados em quatro níveis: Abaixo do Básico, Básico, Adequado e Avançado. O Quadro 1 contém informações referentes aos níveis de proficiência, pontuação, classificação e descrição, utilizados pelo SARESP.

Quadro 1: Classificação e descrição dos níveis de proficiência do SARESP.

\begin{tabular}{|c|c|c|c|}
\hline $\begin{array}{c}\text { Níveis de } \\
\text { Proficiência }\end{array}$ & $\begin{array}{l}\text { Intervalos de } \\
\text { Pontuação }\end{array}$ & Classificação & Descrição \\
\hline $\begin{array}{l}\text { Abaixo do } \\
\text { básico }\end{array}$ & $\begin{array}{l}\text { Menor que } \\
275\end{array}$ & Insuficiente & $\begin{array}{l}\text { Os alunos demonstram domínio } \\
\text { insuficiente dos conteúdos, } \\
\text { competências e habilidades desejáveis } \\
\text { para o ano/série em que se encontram. }\end{array}$ \\
\hline Básico & $\begin{array}{l}\text { Igual ou acima } \\
\text { de } 275 \text { e } \\
\text { abaixo de } 350\end{array}$ & Suficiente & $\begin{array}{l}\text { Os alunos demonstram domínio } \\
\text { mínimo dos conteúdos, competências } \\
\text { e habilidades, mas possuem estruturas } \\
\text { necessárias para interagir com a } \\
\text { proposta curricular no ano/série } \\
\text { subsequente. }\end{array}$ \\
\hline Adequado & $\begin{array}{l}\text { Igual ou acima } \\
\text { de } 350 \text { e } \\
\text { abaixo de } 400\end{array}$ & & $\begin{array}{l}\text { Os alunos demonstram domínio pleno } \\
\text { do conteúdo, competências e } \\
\text { habilidades desejáveis para o ano/série } \\
\text { em que se encontram. }\end{array}$ \\
\hline Avançado & $\begin{array}{l}\text { Igual ou acima } \\
\text { de } 400\end{array}$ & Avançado & $\begin{array}{l}\text { Os alunos demonstram } \\
\text { conhecimentos e domínio dos } \\
\text { conteúdos, competências e habilidades } \\
\text { acima do requerido no ano/série em } \\
\text { que se encontram. }\end{array}$ \\
\hline
\end{tabular}

Fonte: Elaboração da autora a partir do Relatório Pedagógico do SARESP 2011.

${ }^{4}$ As competências cognitivas caracterizam o conjunto de ações e operações mentais utilizado pelo aluno para estabelecer relações com e entre objetos e pessoas.

RIAEE - Revista Ibero-Americana de Estudos em Educação, Araraquara, v.12, n.4, p. 2072-2087, out./set. 2017. 
A primeira coluna do Quadro 1 mostra que os níveis de proficiência dividem-se em quatro categorias: Abaixo do Básico, Básico, Adequado e Avançado. Cada um dos níveis de proficiência possui um intervalo de pontuação, definido a partir da Teoria de Resposta ao Item (ANDRADE E VALLE, 1998), mostrado na segunda coluna. Os níveis de proficiência, combinados com seus intervalos de pontuação, são classificados em um de três grupos, mostrados na terceira coluna, a saber, Insuficiente, Suficiente e Avançado. Deve-se observar que os níveis de proficiência Básico e Adequado são classificados como Suficientes. A quarta coluna contém uma breve descrição de cada nível de proficiência. Conforme a pontuação obtida na prova de matemática, o aluno é classificado em um dos níveis de proficiência, isto é, Insuficiente, Adequado ou Avançado. Vale ressaltar que o padrão de desempenho esperado pelo SARESP é o nível Adequado, o qual inclui os alunos que construíram as habilidades de identificar, calcular e resolver problemas envolvendo média aritmética, proporção, equações e sistemas de equações do $1^{\circ}$ e $2^{\circ}$ graus, progressões aritméticas, medidas, exponenciais e o Teorema de Pitágoras.

\section{Efeitos da quantidade de alunos sobre o desempenho educacional}

O debate sobre a eficácia da redução do tamanho da classe para melhorar o desempenho do aluno tem sido surpreendentemente animado, visto o número de pesquisas realizadas. Tal debate ganhou relevância após incentivos para as reduções no tamanho da classe introduzidas na Califórnia em 1996, resultando em pressões para que o mesmo ocorresse nas escolas de todos os demais estados americanos (HANUSHEK, 1999). No entanto, a literatura mostra que não há um consenso acerca dos impactos da redução do tamanho da classe sobre o desempenho do aluno. Pesquisas sugerem que o tamanho da classe exerce pequenos efeitos ou atuam negativamente sobre os resultados educacionais do indivíduo. Na literatura, os resultados encontrados envoltos dos efeitos da escola sobre o desempenho do aluno são ambíguos, conflitantes e fracos (FINN E ACHILLES, 1990; ANGRIST E LAVY, 1999; URQUIOLA, 2006), evidenciando que a questão do tamanho da sala de aula continua a atrair a atenção dos formuladores de políticas educacionais e pesquisadores. Para o pessoal da escola, pequenas classes garantem facilitar o aumento da interação estudante - professor, permitem uma completa e continua avaliação do aluno, e fornecem maior flexibilidade nas estratégias 
de ensino. Este não é o processo somente desejado, mas também o que beneficia o estudante em risco de insucesso escolar.

Finn e Achilles (1990) descrevem um experimento de larga escala no qual alunos do jardim da infância e professores foram alocados em pequenas e grandes classes dentro de cada escola participante e os resultados mostraram que os estudantes colocados nas salas reduzidas foram beneficiados. Os autores argumentam que tais benefícios são oriundos do fato de que em classes menores a responsabilidade do professor com trabalho administrativo, tal como a manutenção de registros de alunos, é reduzida permitindo que dediquem mais tempo às atividades relevantes. Além disso, em salas de aula pequenas o professor pode manter os alunos sob olhar atento, possibilitando minimizar os problemas de disciplina entre os estudantes, e também aumentar as interações individuais, dedicando mais atenção a cada aluno. No entanto, manter classes com reduzidas quantidades de alunos custa caro e exige investimento em salários de professores e salas de aula adicionais, os quais somente são prováveis se as evidências de que os resultados são positivos forem substanciais e consistentes.

De acordo com Angrist e Lavy (1999, p. 540), "the academic interest and public controversy surrounding the class size question is not only a modern phenomenon; the choice of class size has been of concern to scholars and teachers for hundreds of years". Baseados no fato de que o tamanho da sala de aula sobre o desempenho do aluno tem sido um tema preocupante na literatura educacional, os autores consideraram dados referentes a escolas israelenses para analisar a relação existente entre o número de matrículas e o tamanho da classe. Os autores utilizaram esta relação para construir variáveis instrumentais sobre os escores de testes realizados pelos alunos e, a partir dos resultados obtidos, concluíram que reduções no tamanho da sala resultam em um significante e substancial aumento no aprendizado do aluno no que se refere à leitura e à matemática. Os resultados sugerem que os benefícios proporcionados por pequenas classes são maiores para aqueles alunos com mais dificuldades de aprendizagem.

Krueger (1999) analisou dados de estudantes e seus professores, os quais foram aleatoriamente atribuídos a diferentes tamanhos de classes. Utilizando-se de testes estatísticos, o autor concluiu que, em média, o desempenho em testes padronizados aumenta para os estudantes em pequenas turmas e que a vantagem na pontuação no teste de alunos em turmas pequenas aumenta em cerca de um ponto percentual por ano nos anos seguintes. Os resultados mostraram também que ajudantes e características de 
professores exercem pouco efeito sobre a proficiência do aluno e que o tamanho da classe tem um efeito maior para a minoria dos estudantes.

Urquiola (2006) apresentou duas estratégias para identificar os efeitos do tamanho da classe sobre o desempenho em escolas da Bolívia. Os resultados alcançados mostraram que classes maiores têm um efeito negativo sobre o desempenho dos alunos, isto é, para ambas as estratégias, salas de aula com grandes quantidades de alunos resultam em pontuações baixas nas avaliações realizadas.

Para Hanushek (2002), as evidências sugerem que qualquer efeito sobre a redução do tamanho da classe será mínimo e muito caro. Investigações mostram algum efeito do tamanho da sala sobre o desempenho para um grupo pequeno ou sob determinadas circunstâncias. O autor ressalta que o valor adicionado para turmas menores é praticamente nulo, exceto para os alunos que frequentam o primeiro ano de ensino, mas os efeitos estimados são insuficientes para apoiar qualquer ampliação das políticas de redução. Hanushek (2002) argumenta ainda que as políticas de redução do tamanho da classe geralmente não deixam margem para as localidades decidirem quando e onde tais reduções seriam benéficas ou prejudiciais ao desempenho do aluno.

Os resultados encontrados na literatura não dizem que reduções no tamanho da sala nunca são eficientes ou e que nunca deveriam ser implementadas, mas que, na forma como são apresentadas no debate persistente na literatura, as propostas de redução não são suscetíveis de eficácia no que se refere ao desempenho do aluno.

\section{Análise empírica de correlação: número de alunos por escola $x$ proficiência média em matemática no SARESP}

Tendo a discussão citada anteriormente como ponto de partida, o presente estudo tem por objetivo investigar a existência de uma relação entre a quantidade de alunos por escola, que participaram das edições do SARESP de 2008 e 2013, com o desempenho alcançado em matemática. Foram consideradas as quantidades de alunos e as notas médias dos estudantes da terceira série do Ensino Médio somente das escolas da rede pública estadual de ensino. Os dados utilizados nesta pesquisa foram disponibilizados pela Secretaria Estadual de Educação, especificamente pela Coordenadoria de Informação, Monitoramento e Avaliação Educacional (SEE/CIMA) agrupados conforme as regiões do estado de São Paulo, isto é, Capital, Centro, Litoral, Nordeste, Noroeste, Norte, Sudeste e Sudoeste

RIAEE - Revista Ibero-Americana de Estudos em Educação, Araraquara, v.12, n.4, p. 2072-2087, out./set. 2017. 
De acordo com Menezes (2001), as Diretorias de Ensino (DE) são definidas como unidades da administração direta subordinadas à Secretaria Estadual de Educação e foram criadas com a justificativa de acabar com o desperdício de recursos humanos e promover a aplicação eficaz dos recursos financeiros. No estado de São Paulo há um total de 91 DE, ocupando áreas da Grande São Paulo, da Capital e demais partes. Espalhadas pelo Estado totalizam 63 DE. As escolas contidas na amostra de dados considerada foram classificadas em regiões conforme a localização da DE.

A investigação sobre a existência de relação entre o número de alunos, por escola, que participou do SARESP em 2008 e 2013, com o desempenho alcançado em matemática, inicia-se a partir da elaboração do diagrama de dispersão entre tais variáveis. Tal gráfico ilustra a disposição dos pares ordenados correspondentes às variáveis "quantidade de alunos" e "desempenho em matemática", e proporciona uma ideia, a priori, concomitantemente da existência de uma relação entre tais medidas e da direção desta associação. A Figura 1 contém os diagramas de dispersão para cada uma das regiões do estado de São Paulo.

Os diagramas de dispersão associados ao ano de 2008 evidenciam uma associação linear entre a quantidade de alunos por escola e o desempenho em matemática. Tal relação possui, aparentemente, caráter positivo, pois sugere que, à medida que a quantidade de alunos aumenta, melhores desempenhos são alcançados. Por outro lado, desempenhos mais baixos são alcançados quando menos alunos fazem a avaliação. O mesmo pode ser afirmado para todas as regiões, uma vez que o comportamento dos gráficos é similar; a diferença entre todos está apenas na quantidade de pontos, pois o número de escolas e alunos é maior nas grandes regiões, como Capital e Centro, e menores nas demais, sendo a Litoral a menor dentre todas as outras.

Para os dados observados em 2013, os diagramas de dispersão resultantes também sugerem a existência de uma relação linear positiva entre a quantidade de alunos por escola e o desempenho em matemática. No entanto, para as regiões Capital, Litoral e Sudeste, tal associação é menos acentuada quando comparada às demais. Difere-se dos outros o gráfico correspondente aos dados da região Norte do Estado, a qual apresenta uma tendência decrescente, sugerindo que um aumento na quantidade de alunos resultaria em um desempenho mais baixo.

Após interpretação dos diagramas de dispersão mostrados na Figura 1, os coeficientes de correlação entre a quantidade de alunos por escola e o desempenho em

RIAEE - Revista Ibero-Americana de Estudos em Educação, Araraquara, v.12, n.4, p. 2072-2087, out./set. 2017. 
matemática foram calculados para os anos de 2008 e 2013, separadamente para cada região do estado de São Paulo, a partir da expressão representada por:

$$
r=\frac{n \sum x y-\left(\sum x\right)\left(\sum y\right)}{\sqrt{n \sum x^{2}-\left(\sum x\right)^{2}} \sqrt{n \sum y^{2}-\left(\sum y\right)^{2}}},
$$

Figura 1: Diagramas de dispersão com dados de 2008 e 2013.

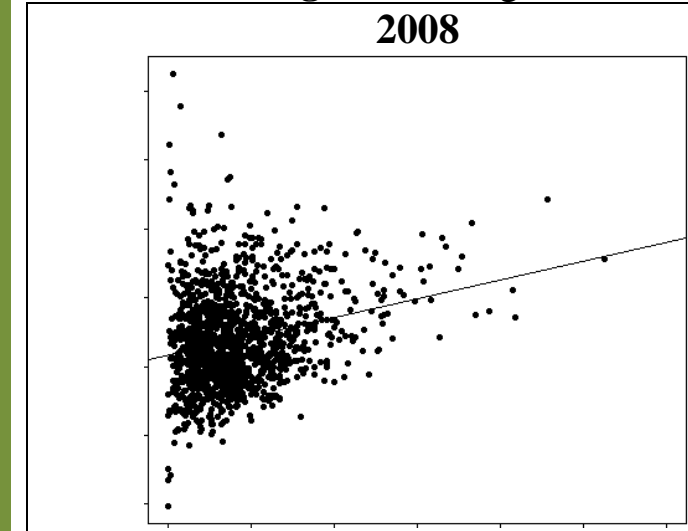

Capital

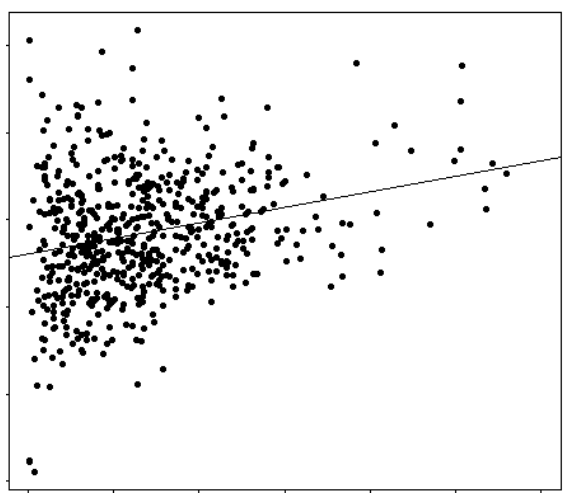

Centro

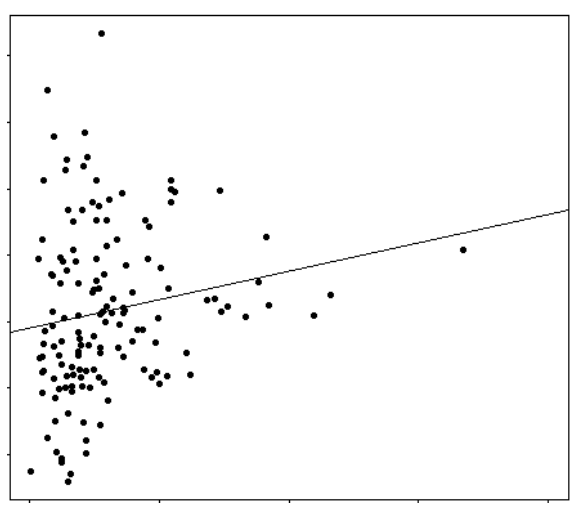

Litoral

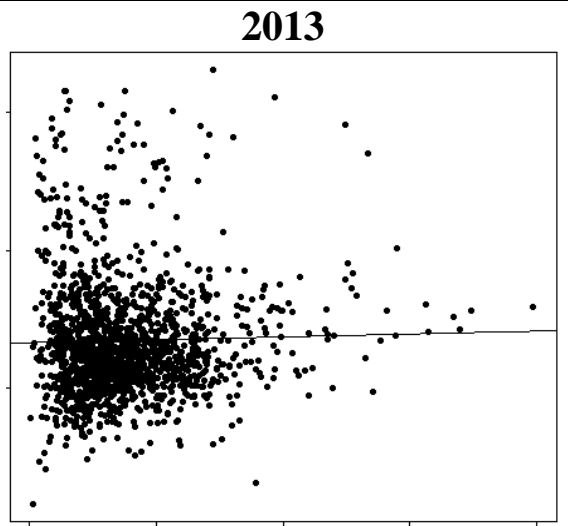

Capital

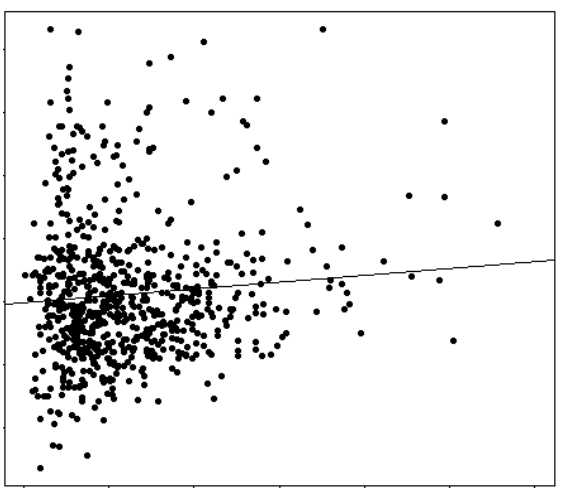

Centro

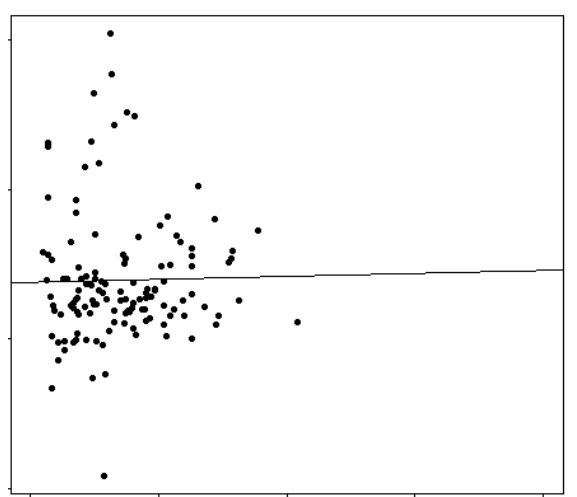

Litoral

RIAEE - Revista Ibero-Americana de Estudos em Educação, Araraquara, v.12, n.4, p. 2072-2087, out./set. 2017. 


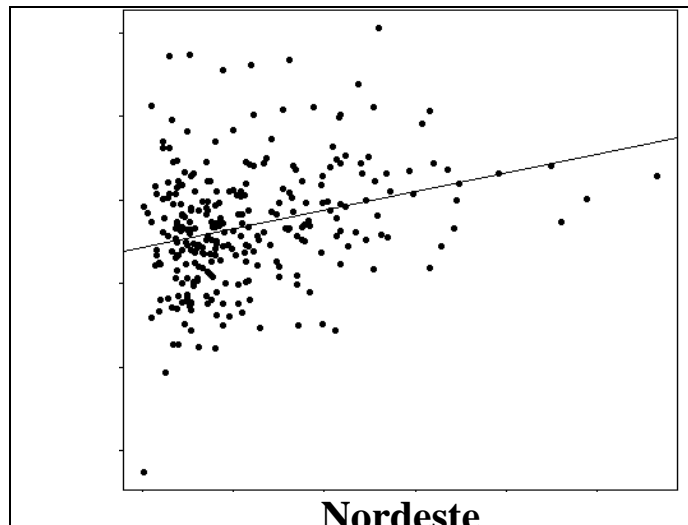

Continua na próxima página.

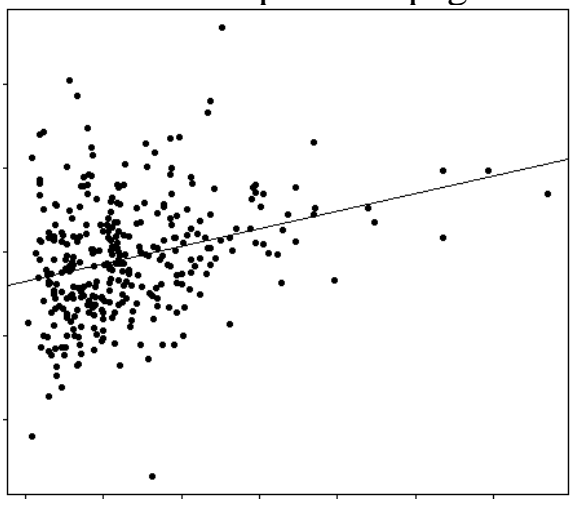

Noroeste

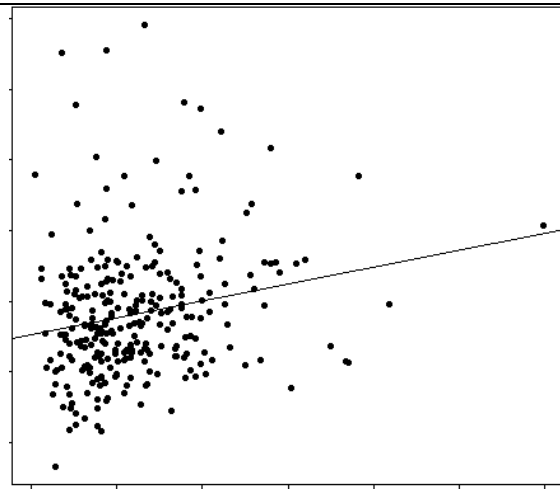

Nordeste

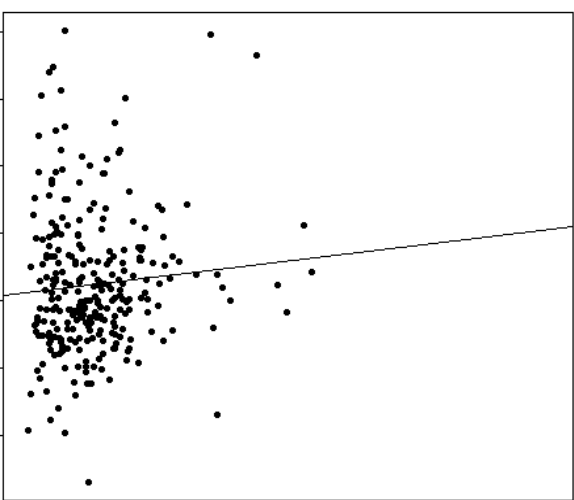

Noroeste

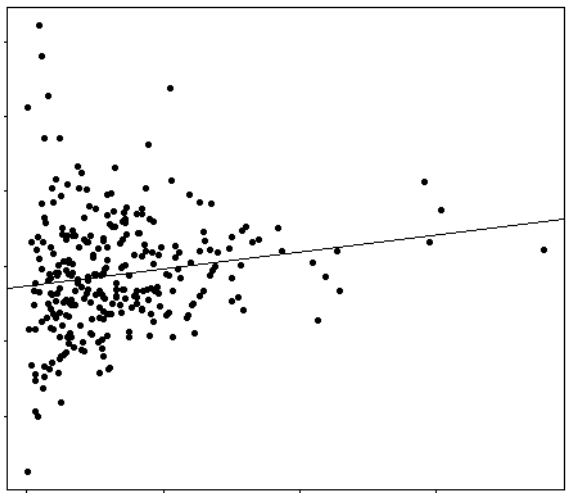

Norte

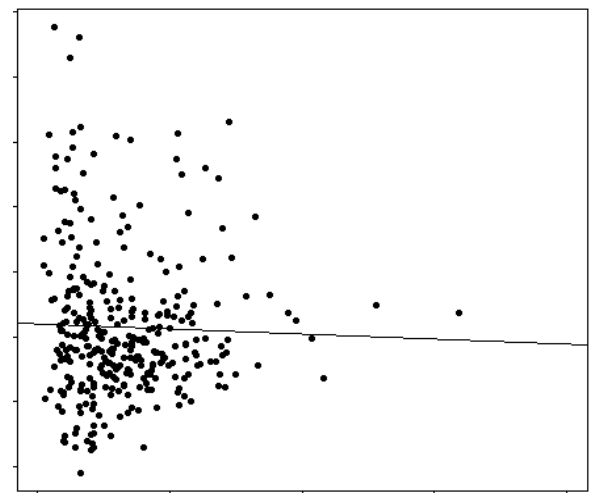

Norte

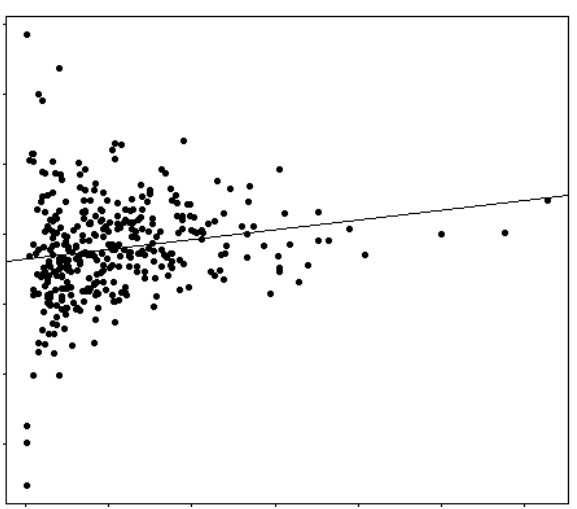

Sudeste

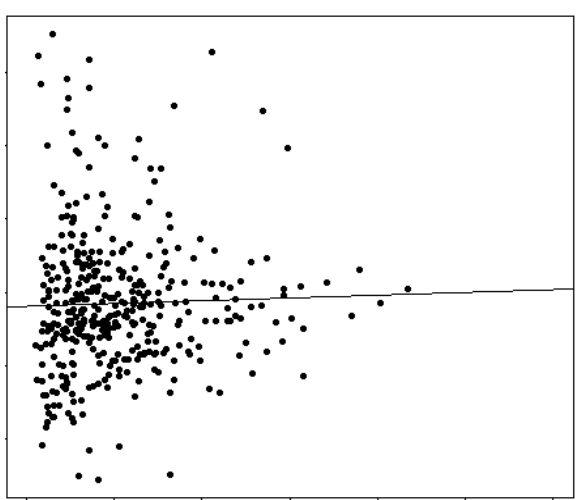

Sudeste

RIAEE - Revista Ibero-Americana de Estudos em Educação, Araraquara, v.12, n.4, p. 2072-2087, out./set. 2017.

E-ISSN: 1982-5587

DOI: 10.21723/riaee.v12.n4.out./dez.2017.10670 


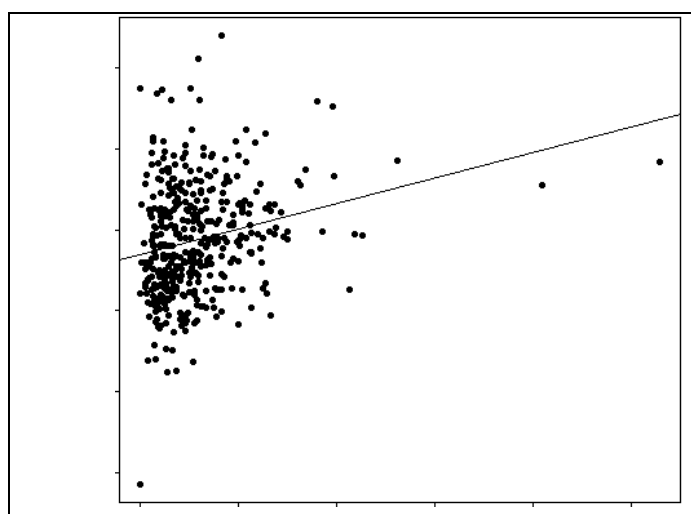

Sudoeste

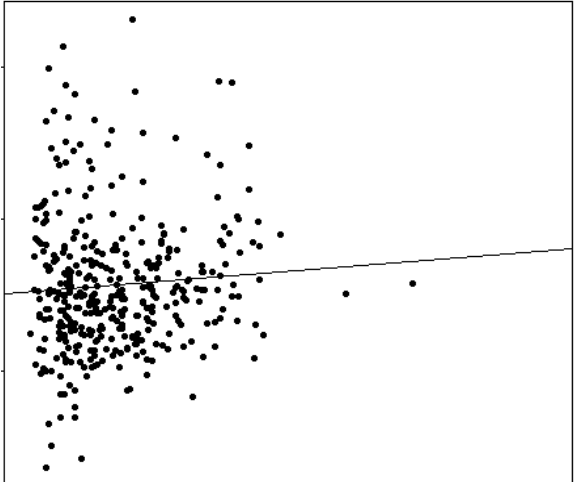

Sudoeste

Fonte: Elaboração própria, a partir dos dados disponibilizados pela CIMA - SEE/SP, e utilizando o software R.

Na expressão (1), $n$ indica o número de observações, $r$ é uma medida da força e direção da relação entre duas variáveis e varia entre 1 e -1 . Enquanto valores de $r$ próximos de 1 indicam uma forte correlação positiva entre as variáveis, valores próximos de -1 sugerem uma relação em direções opostas entre as medidas, isto é, à medida que uma aumenta, a outra diminui. Quando prevalece a ausência de correlação entre as variáveis, o valor de $r$ é próximo de zero (LARSON; FARBER, 2010).

Com o suporte do software R, os coeficientes de correlação foram obtidos para todas as regiões, com dados de 2008 e 2013. Tais valores são mostrados na Tabela 1, juntamente com as quantidades médias de alunos por escola que participaram do SARESP e as notas médias alcançadas em matemática, por região.

A partir dos valores mostrados na Tabela 1 é possível observar que, em 2008, as quantidades de alunos foram maiores na Capital do Estado e nas regiões Noroeste e Sudoeste, quando comparadas com as quantidades registradas em 2013. Nas demais regiões, mais alunos participaram da edição de 2013 do SARESP. Em relação às médias, maiores valores foram registrados em 2008 somente na Capital e na região litorânea do Estado. Já em 2013, médias mais altas foram alcançadas por alunos residentes na região central do Estado e nas demais regiões, isto é, Nordeste, Noroeste, Norte, Sudeste e Sudoeste.

Tabela 1: Proficiência média em matemática, quantidade de alunos por escola e coeficientes de correlação.

\begin{tabular}{lccc|ccc}
\hline \multirow{2}{*}{ Região } & \multicolumn{3}{c|}{$\mathbf{2 0 0 8}$} & \multicolumn{3}{c}{$\mathbf{2 0 1 3}$} \\
\cline { 2 - 7 } & Média & $\mathbf{N}^{\circ}$ alunos & Correlação* & Média & $\mathbf{N}^{\circ}$ alunos & Correlação* \\
\hline Capital & 267,88 & 112.115 & 0,2486 & 267,24 & 110.320 & 0,0263 \\
\hline Centro & 276,83 & 39.625 & 0,2572 & 282,38 & 42.859 & 0,0829 \\
\hline
\end{tabular}




\begin{tabular}{lccc|ccc}
\hline Litoral & 271,69 & 8.430 & 0,1695 & 269,68 & 8.896 & 0,0195 \\
\hline Nordeste & 273,81 & 16.469 & 0,2883 & 276,30 & 16.680 & 0,2038 \\
\hline Noroeste & 278,52 & 21.149 & 0,2909 & 284,74 & 14.743 & 0,0819 \\
\hline Norte & 277,79 & 18.638 & 0,1583 & 283,00 & 20.760 & $-0,0308$ \\
\hline Sudeste & 275,77 & 19.042 & 0,1772 & 277,06 & 19.837 & 0,0295 \\
\hline Sudoeste & 277,51 & 23.076 & 0,2289 & 278,39 & 19.750 & 0,0712 \\
\hline
\end{tabular}

": Todos os valores foram significativos ao nível de $1 \%$.

Fonte: Elaboração própria a partir dos dados disponibilizados pela CIMA - SEE/SP e dos cálculos dos coeficientes de correlação realizados no software R.

Os coeficientes de correlação entre a quantidade de alunos por escola que participaram do SARESP em 2008 e seus respectivos desempenhos, evidenciam a existência de uma relação linear positiva em todas as regiões, sendo o maior valor obtido para os dados correspondentes à região Noroeste, igual a 0,2909. Tal coeficiente de correlação sugere que, à medida que aumenta a quantidade de alunos participantes da avaliação, melhores desempenhos são alcançados, em nível de escola.

Para o ano de 2013, as médias em matemática e as quantidades de alunos que participaram do SARESP foram mais altas nas regiões Centro, Nordeste, Norte e Sudeste, quando comparadas com a edição de 2008, sugerindo que escolas com mais alunos participando da avaliação terão melhores desempenhos. Porém, as médias registradas nas regiões Noroeste e Sudoeste foram mais altas em 2013, mas com quantidades menores de alunos, o que impossibilita generalizar a conclusão de que, quanto mais alunos nas escolas, melhores serão os resultados na avaliação educacional. Em relação aos coeficientes de correlação, embora significativos, os valores foram mais baixos, comparados àqueles obtidos em 2008. Os valores dos coeficientes de correlação para o ano de 2013 pertencem ao intervalo [-0,0308;0,2038], sendo o mais alto registrado na região Nordeste e o mais baixo na região Norte. Com um coeficiente de correlação negativo na região Norte, as evidências apontam que, à medida que aumenta a quantidade de alunos, pior será o desempenho em matemática, corroborando com as conclusões dos estudos de Finn e Achilles (1990) e de Urquiola (2006).

Os resultados obtidos a partir da análise de correlação entre a quantidade de alunos por escola e o respectivo desempenho médio em matemática, considerando dados do SARESP de 2008 e 2013, por região do estado de São Paulo, são diversos e distintos entre si. Os coeficientes de correlação obtidos mostram que, enquanto há regiões com maiores quantidades de alunos que participaram da avaliação e alcançaram

RIAEE - Revista Ibero-Americana de Estudos em Educação, Araraquara, v.12, n.4, p. 2072-2087, out./set. 2017. 
melhores desempenhos em um ano, quando comparado com outro, também há regiões onde menos alunos proporcionaram melhores resultados para a escola. Valores positivos e negativos foram encontrados para tal coeficiente, sugerindo que um aumento no número de aluno, ora gera melhores resultados, ora piores. Tal observação é similar aos resultados encontrados na literatura que investiga os impactos do tamanho das salas de aula sobre o desempenho educacional, isto é, de que não há um consenso acerca da associação entre tais variáveis.

\section{Considerações finais}

A questão educacional perpassa muitos aspectos, como necessidades de recursos e financiamentos, projetos político-pedagógicos, gestão e administração por parte de estados e municípios, enfim, de ações vindas das políticas públicas em sentido amplo, com suas prioridades e diretrizes. Em geral, os objetivos dos planejamentos na área educacional levam em conta as demandas da escolarização para a população em idade escolar ideal. Medir o desempenho educacional e compreender seus determinantes caracterizam ações importantes para a concepção de políticas em relação a esses temas variados. O presente estudo procura melhor compreensão de fatores que influenciam diretamente o trabalho em sala de aula e o número de alunos por escola é um desses fatores. De que forma e até onde essa componente influencia no desempenho? A partir da amostra considerada e dos resultados obtidos com a análise de correlação entre o número médio de alunos por escola e a pontuação média alcançada em matemática nas edições do SARESP de 2008 e 2013. Os diagramas de dispersão e valores dos coeficientes de correlação, calculados por região do estado de São Paulo, permitiram observar que há correlações moderadas positivas entre o número médio de alunos por escola e a pontuação média obtida e há, também, correlação considerada fraca que também sugere que escolas com mais alunos têm desempenho mais baixo. Nessa amostra há também uma região cujo resultado sugere que escolas maiores no número de alunos participantes da avaliação geram médias em matemática mais altas. Há ainda regiões cujos coeficientes de correlação indicam a ausência de uma relação linear entre as variáveis, onde a pontuação média na avaliação não é, pela análise da correlação, influenciada pela quantidade de alunos que participaram da avaliação.

Dada a complexidade das variáveis envolvidas nesse tipo de estudo, há inúmeras controvérsias sobre a existência de um efeito contínuo sobre a correlação entre o 
número médio de alunos por escola que fazem a avaliação e a pontuação média obtida em matemática, assim como a dimensão de sua influência, ou ainda se o mais provável é o pertencimento a grupos identificados por idade, sexo, origem étnica ou habilidades.

AGRADECIMENTOS: Os autores são gratos à Fundação de Amparo à Pesquisa do Estado de São Paulo - FAPESP, pelo suporte financeiro e à Coordenadoria de Informação, Monitoramento e Avaliação Educacional - CIMA pela disponibilização dos dados utilizados no presente estudo.

\section{REFERÊNCIAS}

ANDRADE, Dalton Francisco de.; VALLE, Raquel da Cunha. Introdução à Teoria de Resposta ao Item. Estudos em Avaliação Educacional, n. 18, p. 1-20, 1998.

ANGRIST, Joshua.; LAVY, Victor. Using Maimonides' Rule to Estimate the Effect of Class Size on Scholastic Achievement. Quarterly Journal of Economics, v. 114, n. 2, p. 533-575, 1999.

BARBOSA, Maria Eugenia Ferrão; FERNANDES, Cristiano. A Escola Brasileira Faz Diferença? Uma Investigação dos Efeitos da Escola na Proficiência em Matemática dos Alunos da $4^{a}$ série. In: CRESO, Franco. Avaliação, Ciclos e Promoção na Educação. Porto Alegre: Artmed Editora, 2001.

BRASIL. Secretaria da Educação do Estado de São Paulo. Relatório Pedagógico SARESP 2011. São Paulo, SP: SEE, 2011.

FINN, Jeremy.; ACHILLES, Charles. Answers and Questions about Class Size: A Statewide Experiment. American Educational Research Journal, v. 27, n. 3, p. 557 577, 1990.

HANUSHEK, Eric. Some findings from an independent investigation of the Tennessee star experiment and from other investigations of class size effects, Educational Evaluation and Policy Analysis, v. 21, n. 2, p. 143-164, 1999.

HANUSHEK, Eric. Evidence, politics, and the class size debate. In: MISHEL, Lawrence.; ROTHSTEIN, Richard (Orgs.). The Class Size Debate. Washington, D.C.: Economic Policy Institute, 2002. P. 37-66.

JESUS, Girlene Ribeiro de.; LAROS, Jacob Arie. Eficácia escolar: Regressão Multinível com Dados de Avaliação em Larga Escala. Avaliação Psicológica, v. 3, n. 2 , p. 93-106, 2004.

KRUEGER, Alan. Experimental Estimates of Education Production Functions.

Quarterly Journal of Economics, v. 114, n. 2, p. 497-531, 1999.

RIAEE - Revista Ibero-Americana de Estudos em Educação, Araraquara, v.12, n.4, p. 2072-2087, out./set. 2017. 
LARSON, Ron.; FARBER, Betsy. Estatística Aplicada. 4ª ed. Prentice Hall Brasil, 2010. $656 \mathrm{p}$.

MENEZES, Ebenezer Takuno de.; SANTOS, Thais Helena dos. Verbete Diretorias de ensino. Dicionário Interativo da Educação Brasileira - Educabrasil. São Paulo:

Midiamix, 2001. Disponível em: <http://www.educabrasil.com.br/diretorias-deensino/>. Acesso em: 8 maio 2017.

SOARES, José Francisco.; COLLARES, Ana Cristina Murta. Recursos Familiares e o Desempenho Cognitivo dos Alunos do Ensino Básico Brasileiro. DADOS - Revista de Ciências Sociais, Rio de Janeiro, v. 49, n. 3, p. 615 a 481, 2006.

URQUIOLA, Miguel. Identifying Class Size Effects in Developing Countries: Evidence from Rural Bolivia. The Review of Economics and Statistics, v. 88, n. 1, p. 171 - 177, 2006.

\section{Como referenciar este artigo}

BASSETTO, Camila Fernanda.; FERREIRA, Eric Chibana.; AGUIAR, Renato Forte. Relação entre desempenho educacional e quantidade de alunos: uma análise empírica regional com dados do SARESP. Revista Ibero-Americana de Estudos em Educação, Araraquara, v. 12, n. 4, p. 2072-2087, out./dez. 2017. Disponível em: <http://dx.doi.org/10.21723/riaee.v12.n4.out./dez.2017.10670>. E-ISSN: 1982-5587.

Submetido em: 16/09/2017

Revisões requeridas: 18/10/2017

Aceite em: 11/11/2017 